\title{
Identifying an Unknown Coefficient in the Reaction-Diffusion Equation Using He's VIM
}

\author{
F. Parzlivand and A. M. Shahrezaee \\ Department of Mathematics, Alzahra University, Vanak, Tehran 19834, Iran \\ Correspondence should be addressed to A. M. Shahrezaee; ashahrezaee@alzahra.ac.ir
}

Received 16 July 2014; Accepted 2 October 2014; Published 19 October 2014

Academic Editor: Delin Chu

Copyright ( 2014 F. Parzlivand and A. M. Shahrezaee. This is an open access article distributed under the Creative Commons Attribution License, which permits unrestricted use, distribution, and reproduction in any medium, provided the original work is properly cited.

\begin{abstract}
An inverse heat problem of finding an unknown parameter $p(t)$ in the parabolic initial-boundary value problem is solved with variational iteration method (VIM). For solving the discussed inverse problem, at first we transform it into a nonlinear direct problem and then use the proposed method. Also an error analysis is presented for the method and prior and posterior error bounds of the approximate solution are estimated. The main property of the method is in its flexibility and ability to solve nonlinear equation accurately and conveniently. Some examples are given to illustrate the effectiveness and convenience of the method.
\end{abstract}

\section{Introduction}

The parameter determination in a parabolic partial differential equation from the overspecified data plays a crucial role in applied mathematics and physics. This technique has been widely used to determine the unknown properties of a region by measuring data only on its boundary or a specified location in the domain. These unknown properties, such as the conductivity medium, are important to the physical process, but they usually cannot be measured directly, or the process of their measurement is very expensive [1]. These problems are often ill-posed [2] and a direct inversion of the data for the unknown function is not possible. As a result, a number of researchers have developed various methods to overcome the ill-posed nature of the inversion problem. These methods include Born approximation [3], neuralnetworks [4], and Levenberg-Marquardt method [5].

In this paper, we solve an inverse problem to a class of reaction-diffusion equation using variational iteration method. The method is capable of reducing the size of calculations and handles both linear and nonlinear equations, homogeneous or inhomogeneous, in a direct manner. The method gives the solution in the form of a rapidly convergent successive approximation that may give the exact solution if such a solution exists. For concrete problems where exact solution is not obtainable, it was found that a small number of approximations can be used for numerical purposes.

1.1. Reaction-Diffusion Systems. Reaction-diffusion systems are mathematical models which explain how the concentration of one or more substances distributed in space changes under the influence of two processes: local chemical reactions in which the substances are transformed into each other and diffusion which causes the substances to spread out over a surface in space. This description implies that reaction-diffusion systems are naturally applied in chemistry. However, the system can also describe dynamical processes of nonchemical nature. Examples are found in biology, geology, physics, and ecology [6, 7]. Mathematically, reactiondiffusion systems take the form of semilinear parabolic partial differential equations. They can be represented in the general form:

$$
u_{t}=\nabla \cdot(D(x, t, u) \nabla u)+f(u, \nabla u ; x, t),
$$

where $u=u(x, t)$ represents the concentration of one substance, $D$ is a diffusion coefficient, and $f$ is the reaction term.

1.2. A Class of Reaction-Diffusion Systems. In this paper, we consider an inverse problem of simultaneously finding 
unknown coefficients $p(t)$ and $u(x, t)$ satisfying reactiondiffusion equation

$$
u_{t}=a(t) u_{x x}+p(t) u_{x}+f(x, t) ; \quad \text { in } Q_{T},
$$

with the initial-boundary conditions

$$
\begin{array}{ll}
u(x, 0)=u_{0}(x) ; & 0 \leq x \leq 1, \\
B_{1} u(0, t)=g_{0}(t) ; & 0 \leq t \leq T, \\
B_{2} u(1, t)=g_{1}(t) ; & 0 \leq t \leq T,
\end{array}
$$

where $u(x, t)$ is the concentration, $a(t)$ is a diffusion coefficient, $p(t) u_{x}(x, t)+f(x, t)$ is the reaction term, $Q_{T}=$ $\{(x, t), 0<x<1,0<t<T\}, T>0, B_{1}$ and $B_{2}$ are boundary operators (i.e., $B_{1}, B_{2}=\partial^{i} / \partial x^{i}, i=0$ or 1), and $a, f, u_{0}, g_{0}$, and $g_{1}$ are known functions.

An additional boundary condition which can be the integral overspecification is given in the following form:

$$
\int_{0}^{s(t)} k(x) u(x, t) d x=E(t) ; \quad 0<t \leq T, 0<s(t)<1,
$$

where $E, s$, and $k$ are known functions and for some $\rho>0$, the kernel $k(x)$ satisfies

$$
\int_{0}^{1}|k(x)| d x \leq \rho .
$$

Without the extra measurement (4), problem (2)-(3) is underdetermined and may have many solutions infinitely. On the other hand, if too many additional conditions are imposed, the solution may not exist. The existence and uniqueness of the solution of this problem and more applications and background of the problem are discussed in [8-10]. Also, to interpret integral equation (4), the reader can refer to [11].

1.3. A Brief Discussion on Some Inverse Problems. Inverse problems in partial differential equations can be used to model many real problems in engineering and other physical sciences (cf. [12, 13] for examples). Studying these problems has a great deal of importance both theoretically and practically. We give a quick review of the previous work related to our problems. For the inverse problem (2)-(3) of simultaneously finding unknown coefficients $a(t)$ and $u(x, t)$ with $p(t)=f(x, t)=0$ and additional condition

$$
a(t) u_{x}(0, t)=E(t) ; \quad 0 \leq t \leq T .
$$

Jones [14] obtains the global solvability via an integral equation and Schauder fixed point theorem. Moreover, in [15] the numerical solution by the finite difference method is discussed. Similar problems in different regions are also treated in $[16,17]$. By employing the heat potential and Green's representation, the authors in [18] give the explicit solution for inverse problem of finding $f(t)$ in the equation

$$
u_{t}=u_{x x}+f(t) \text {. }
$$

These examples motivate us to consider the following general problem:

$$
u_{t}=a\left(x, t, u, u_{x}, u_{x}\left(x^{\star}, t\right)\right) u_{x x}+b\left(x, t, u, u_{x}, u_{x}\left(x^{\star}, t\right)\right),
$$

subject to initial-boundary conditions (3), where $0<x^{\star}<1$ is a fixed point. The global solvability for this problem is established in [19].

This paper is organized as follows. In Section 2, the variational iteration method is recapitulated. In Section 3, an error analysis is presented for the proposed method and prior and posterior error bounds of the approximate solution are estimated. In Section 3, at first we transform the inverse problem (2)-(4) into a nonlinear direct problem and then the VIM is used for solving this problem. To present a clear overview of the new method, some examples are given in Section 4. A conclusion is presented in the last section.

\section{Basic Idea of the Variational Iteration Method}

The variational iteration method is a powerful tool to search for both analytical and approximate solutions of nonlinear equation without requirement of linearization or perturbation [20, 21]. The method was first proposed by He in 1998 [22]. Also it was successfully applied to various engineering problems [23-27]. In 1978, Inokuti et al. [28] proposed a general Lagrange multiplier method to solve nonlinear problems, which was first proposed to solve problems in quantum mechanics (see [28] and the references cited therein). The main feature of the method is as follows: the solution of a mathematical problem with linearization assumption is used as initial approximation or trial-function; then a more highly precise approximation at some special point can be obtained.

Consider the following general nonlinear system:

$$
L u(t)+N u(t)=f(t),
$$

where $L$ and $N$ are linear and nonlinear operators, respectively, and $f$ is source or sink term.

Assuming $u_{0}(t)$ is the solution of $L u=0$, according to [28], we can write down an expression to correct the value of some special point, for example, at $t=1$ :

$$
u_{\text {cor }}(1)=u_{0}(1)+\int_{0}^{1} \lambda(\tau)\left\{L u_{0}(\tau)+N u_{0}(\tau)-f(\tau)\right\} d \tau,
$$

where $\lambda$ is general Lagrange multiplier [28], which can be identified optimally via the variational theory [29]. The second term on the right is called the correction. He in 1998 [22] modified the above method into an iteration method [20-22] in the following way:

$u_{n+1}\left(t_{0}\right)=u_{n}\left(t_{0}\right)+\int_{0}^{t_{0}} \lambda(\tau)\left\{L u_{n}(\tau)+N \tilde{u}_{n}(\tau)-f(\tau)\right\} d \tau$.

The subscript $n$ denotes the $n$th order approximation and $\tilde{u}_{n}$ is considered as restricted variation [29] which means $\delta \tilde{u}_{n}=0$. 
For arbitrary $t_{0}$, we can rewrite the above equation as follows:

$$
\begin{aligned}
u_{n+1}(t)= & u_{n}(t) \\
& +\int_{0}^{t} \lambda(\tau)\left\{L u_{n}(\tau)+N \widetilde{u}_{n}(\tau)-f(\tau)\right\} d \tau ; \quad n \geqslant 0 .
\end{aligned}
$$

Equation (12) is called a correction functional. It is required first to determine the Lagrange multiplier. Employing the restricted variation in correction functional and using integration by part make it easy to compute the Lagrange multiplier; see, for instance, [30]. For linear problems, its exact solution can be obtained by only one iteration step due to the fact that no nonlinear operator exists so the Lagrange multiplier can be exactly identified. Having $\lambda$ determined, then several approximations $u_{n+1}(t), n \geqslant 0$, can be determined. Under reasonable choice of $u_{0}$, the fixed point of the correction functional is considered as an approximate solution of the above general differential equation. Generally one iteration leads to high accurate solution by variational iteration method if the initial solution is carefully chosen with some unknown parameters. Comparison of the method with Adomian method was conducted by many authors via illustrative examples, and especially Wazwaz gave a complete comparison between the two methods [31], revealing the variational iteration method has many merits over Adomian method: it can completely overcome the difficulty arising in the calculation of Adomian polynomial.

\section{Convergence Analysis and Error Bound of VIM}

This section covers the error analysis of the proposed method. Also the sufficient conditions are presented to guarantee the convergence of VIM, when applied to solve the differential equations.

3.1. Convergence Analysis. First, we will rewrite (12) in the operator form as follows:

$$
u_{n+1}=A\left[u_{n}\right]
$$

where the operator $A$ takes the following form:

$$
A[u(t)]=u(t)+\int_{0}^{t} \lambda(\tau)\{L u(\tau)+N u(\tau)-f(\tau)\} d \tau
$$

Theorem 1. Let $(X,\|\cdot\|)$ be a Banach space and $A: X \rightarrow X$ is a nonlinear mapping and suppose that

$$
\|A[u]-A[\tilde{u}]\| \leq \gamma\|u-\tilde{u}\|, \quad u, \tilde{u} \in X,
$$

for some constant $\gamma<1$. Then, A has a unique fixed point. Furthermore, sequence (12) using VIM with an arbitrary choice of $u_{0} \in X$ converges to the fixed point of $A$ and

$$
\left\|u_{n}-u_{m}\right\| \leq\left\|u_{1}-u_{0}\right\| \sum_{j=m-1}^{n-2} \gamma^{j} .
$$

Proof (see [32]). According to the above theorem, a sufficient condition for the convergence of the variational iteration method is strictly contraction of $A$. Furthermore, sequence (12) converges to the fixed method of $A$, which is also the solution of (9). Also, the rate of convergence depends on $\gamma$.

3.2. Approximation Error. In the following theorem, we introduce an estimation of the error of the approximate solution of problem (9) and prior and posterior error bounds of the approximate solution are estimated. The prior error bound can be used at the beginning of a calculation for estimating the number of steps necessary to obtain a given accuracy and the posterior error bound can be used at intermediate stages or at the end of a calculation.

Theorem 2. Under the conditions of Theorem 1, error estimates are the prior estimate

$$
\left\|u-u_{n}\right\| \leq \frac{\gamma^{n}}{1-\gamma}\left\|u_{1}-u_{0}\right\|
$$

and the posterior estimate

$$
\left\|u-u_{n}\right\| \leq \frac{\gamma}{1-\gamma}\left\|u_{n}-u_{n-1}\right\|
$$

Also, if one supposes that $A[0]=0$ then the error of the approximate solution $u_{n}$ to problem (9) can be obtained as follows:

$$
\left\|u-u_{n}\right\| \leq \frac{1+\gamma}{1-\gamma} \gamma^{n}\left\|u_{0}\right\|
$$

Proof. Consider

$$
\begin{aligned}
\left\|u_{n+1}-u_{n}\right\| & =\left\|A\left[u_{n}\right]-A\left[u_{n-1}\right]\right\| \\
& \leq \gamma\left\|u_{n}-u_{n-1}\right\| \\
& \leq \gamma^{2}\left\|u_{n-1}-u_{n-2}\right\| \\
& \vdots \\
& \leq \gamma^{n}\left\|u_{1}-u_{0}\right\| .
\end{aligned}
$$

Therefore, for any $m>n$, we have

$$
\begin{aligned}
\left\|u_{m}-u_{n}\right\| \leq & \left\|u_{m}-u_{m-1}\right\| \\
& +\left\|u_{m-1}-u_{m-2}\right\|+\cdots+\left\|u_{n+1}-u_{n}\right\| \\
\leq & \gamma^{m-1}\left\|u_{1}-u_{0}\right\|+\gamma^{m-2}\left\|u_{1}-u_{0}\right\| \\
& +\cdots+\gamma^{n}\left\|u_{1}-u_{0}\right\| \\
= & \left(\gamma^{m-1}+\gamma^{m-2}+\cdots+\gamma^{n}\right)\left\|u_{1}-u_{0}\right\| \\
= & \gamma^{n} \frac{1-\gamma^{m-n}}{1-\gamma}\left\|u_{1}-u_{0}\right\| .
\end{aligned}
$$


Since $0<\gamma<1$, in the numerator, we have $1-\gamma^{m-n}<1$. Consequently,

$$
\left\|u_{m}-u_{n}\right\| \leq \frac{\gamma^{n}}{1-\gamma}\left\|u_{1}-u_{0}\right\| ; \quad m>n .
$$

The first statement follows from (22) by using Theorem 1 , as $m \rightarrow \infty$. We derive (18). Taking $n=1$ and writing $v_{0}$ for $u_{0}$ and $v_{1}$ for $u_{1}$, we have from (17)

$$
\left\|v_{1}-u\right\| \leq \frac{\gamma}{1-\gamma}\left\|v_{1}-v_{0}\right\| .
$$

Setting $v_{0}=u_{n-1}$, we have $v_{1}=A\left[v_{0}\right]=u_{n}$ and obtain (18).

Also, we have

$$
\begin{aligned}
\left\|u_{1}-u_{0}\right\| & \leq\left\|\int_{0}^{t} \lambda(\tau)\left\{L u_{0}(\tau)+N u_{0}(\tau)-f(\tau)\right\} d \tau\right\| \\
& =\left\|A\left[u_{0}\right]-u_{0}\right\| \leq\left\|A\left[u_{0}\right]\right\|+\left\|u_{0}\right\| .
\end{aligned}
$$

We can write

$$
\left\|A\left[u_{0}\right]\right\|=\left\|A\left[u_{0}\right]-A[0]\right\| \leq \gamma\left\|u_{0}\right\| .
$$

Therefore

$$
\left\|u_{1}-u_{0}\right\| \leq(\gamma+1)\left\|u_{0}\right\| .
$$

From Theorem 1 , as $m \rightarrow \infty$, then $u_{m} \rightarrow u$ and by using (22)-(26), inequality (19) can be obtained.

\section{The Application of VIM in Inverse Parabolic Problem (2)-(4)}

To use the variational iteration method for solving the problem (2)-(4), at first we use the following transformation.

4.1. The Employed Transformation. By differentiation with respect to the variable $t$ in (4), one obtains

$$
E^{\prime}(t)=s^{\prime}(t) k(s(t)) u(s(t), t)+\int_{0}^{s(t)} k(x) u_{t}(x, t) d x .
$$

Substituting (2) into the above equation yields

$$
\begin{aligned}
E^{\prime}(t)= & s^{\prime}(t) k(s(t)) u(s(t), t) \\
& +\int_{0}^{s(t)} k(x)\left[u_{x x}(x, t)+p(t) u_{x}(x, t)+f(x, t)\right] d x,
\end{aligned}
$$

and it follows that

$$
\begin{aligned}
p(t)= & \left(E^{\prime}(t)-s^{\prime}(t) k(s(t)) u(s(t), t)\right. \\
& \left.\quad-\int_{0}^{s(t)} k(x)\left[u_{x x}(x, t)+f(x, t)\right] d x\right) \\
& \times\left(\int_{0}^{s(t)} k(x) u_{x}(x, t) d x\right)^{-1},
\end{aligned}
$$

provided that, for any $t \in[0, T], \int_{0}^{s(t)} k(x) u_{x}(x, t) d x$ and $\int_{0}^{s(t)} k(x)\left[u_{x x}(x, t)+f(x, t)\right] d x$ are exit; also $\int_{0}^{s(t)} k(x) u_{x}$ $(x, t) d x \neq 0$.

Therefore, the inverse parabolic problem (2)-(4) is equivalent to the following nonlocal parabolic equation:

$$
\begin{aligned}
u_{t}= & u_{x x} \\
& +\left(E^{\prime}(t)-s^{\prime}(t) k(s(t)) u(s(t), t)\right. \\
& \left.\quad-\int_{0}^{s(t)} k(x)\left[u_{x x}(x, t)+f(x, t)\right] d x\right) \\
& \times\left(\int_{0}^{s(t)} k(x) u_{x}(x, t) d x\right)^{-1} u_{x} \\
& +f(x, t) ; \quad \text { in } Q_{T},
\end{aligned}
$$

with the initial-boundary conditions

$$
\begin{array}{cc}
u(x, 0)=u_{0}(x) ; & 0 \leq x \leq 1, \\
B_{1} u(0, t)=g_{0}(t) ; & 0 \leq t \leq T, \\
B_{2} u(1, t)=g_{1}(t) ; & 0 \leq t \leq T .
\end{array}
$$

Therefore, for solving the inverse problem (2)-(4), we will investigate the direct problem (30)-(31).

4.2. Application. In order to solve problem (30)-(31) using VIM, we can write the following correction functional:

$$
\begin{aligned}
& u_{n+1}(x, t) \\
& =u_{n}(x, t) \\
& +\int_{0}^{t} \lambda(t, \tau) \\
& \times\left\{u_{n \tau}(x, \tau)-\tilde{u}_{n x x}(x, \tau)\right. \\
& -\left(E^{\prime}(\tau)-s^{\prime}(\tau) k(s(\tau)) u_{n}(s(\tau), \tau)\right. \\
& \left.-\int_{0}^{s(\tau)} k(x)\left[u_{n x x}(x, \tau)+f(x, \tau)\right] d x\right) \\
& \times\left(\int_{0}^{s(\tau)} k(x) u_{n x}(x, \tau) d x\right)^{-1} \\
& \left.\times \tilde{u}_{n x}(x, \tau)-f(x, \tau)\right\} d \tau .
\end{aligned}
$$


Making the above correction functional stationary, note that $\delta u_{n}(0)=0$; we have

$$
\begin{aligned}
& \delta u_{n+1}(x, t) \\
& =\delta u_{n}(x, t) \\
& +\delta \int_{0}^{t} \lambda(t, \tau) \\
& \times\left\{u_{n \tau}(x, \tau)-\widetilde{u}_{n x x}(x, \tau)\right. \\
& -\left(E^{\prime}(\tau)-s^{\prime}(\tau) k(s(\tau)) u_{n}(s(\tau), \tau)\right. \\
& \left.-\int_{0}^{s(\tau)} k(x)\left[u_{n x x}(x, \tau)+f(x, \tau)\right] d x\right) \\
& \times\left(\int_{0}^{s(\tau)} k(x) u_{n x}(x, \tau) d x\right)^{-1} \\
& \left.\times \tilde{u}_{n x}(x, \tau)-f(x, \tau)\right\} d \tau .
\end{aligned}
$$

Thus, its stationary condition can be obtained as follows:

$$
\begin{aligned}
\lambda^{\prime}(t, \tau) & =0, \\
1+\left.\lambda(t, \tau)\right|_{\tau=t} & =0 ;
\end{aligned}
$$

therefore $\lambda(t, \tau)=-1$.

Now, the following iteration formula can be obtained as

$$
\begin{aligned}
& u_{n+1}(x, t) \\
& =u_{n}(x, t) \\
& -\int_{0}^{t}\left\{u_{n \tau}(x, \tau)-u_{n x x}(x, \tau)\right. \\
& -\left(E^{\prime}(\tau)-s^{\prime}(\tau) k(s(\tau)) u_{n}(s(\tau), \tau)\right. \\
& \left.-\int_{0}^{s(\tau)} k(x)\left[u_{n x x}(x, \tau)+f(x, \tau)\right] d x\right) \\
& \times\left(\int_{0}^{s(\tau)} k(x) u_{n x}(x, \tau) d x\right)^{-1} u_{n x}(x, \tau) \\
& -f(x, \tau)\} d \tau
\end{aligned}
$$

Here, according to Adomian method, we choose its initial approximate solution as $u_{0}(x, t)=u(x, 0)$.

Having $u=\lim _{n \rightarrow \infty} u_{n}$ determined, then the value of $p(t)$ can be computed by using (29).

\section{Illustrative Examples}

In this section three examples are presented to demonstrate the applicability and accuracy of the method. These tests are chosen such that their analytical solutions are known. But the method developed in this research can be applied to more complicated problems. The numerical implementation is carried out in Microsoft Maple13.

Example 1. We consider the following inverse problem:

$$
\begin{gathered}
u_{t}=u_{x x}+p(t) u_{x}+4 t\left(\frac{1}{2}+2 x\right) ; \quad 0<x<1,0<t<1, \\
u(x, 0)=\left(\frac{1}{2}-x\right) x ; \quad 0 \leq x \leq 1, \\
u(0, t)=2 t^{2}-2 t ; \quad 0 \leq t \leq 1, \\
u_{x}(1, t)=\frac{-3}{2} ; \quad 0 \leq t \leq 1, \\
\int_{0}^{1} u(x, t) d x=2 t^{2}-2 t-\frac{1}{12}, \quad 0 \leq t \leq 1 .
\end{gathered}
$$

The true solution is $u(x, t)=(1 / 2-x) x+2 t^{2}-2 t$ while $p(t)=4 t$. Let $u_{0}(x, t)=u(x, 0)=(1 / 2-x) x$. From (35), we obtain $u_{1}(x, t)=(1 / 2-x) x+2 t^{2}-2 t$, which is the exact solution. Also from (29), we have $p(t)=4 t$, which is equal to the exact $p(t)$ of this example.

Example 2. We consider the inverse problem (2)-(4) as follows:

$$
\begin{gathered}
u_{t}=u_{x x}+p(t) u_{x} \\
+4 e^{4 t}\left(\frac{3}{2}+2 x\right) ; \quad 0<x<1, \quad 0<t<1, \\
u(x, 0)=\left(\frac{1}{2}-x\right) x+2 ; \quad 0 \leq x \leq 1, \\
u(0, t)=2 e^{4 t}-2 t ; \quad 0 \leq t \leq 1, \\
u_{x}(1, t)=\frac{-3}{2} ; \quad 0 \leq t \leq 1, \\
\int_{0}^{1} x u(x, t) d x=e^{4 t}-t, \quad 0 \leq t \leq 1 .
\end{gathered}
$$

The exact solution for this example is $u(x, t)=(1 / 2-x) x+$ $2 e^{4 t}-2 t$ while $p(t)=4 e^{4 t}$. Let $u_{0}(x, t)=u(x, 0)=(1 / 2-$ $x) x+2$.

Using (35) and (29), we obtain $u_{1}(x, t)=(1 / 2-x) x+2 e^{4 t}-$ $2 t$ and $p(t)=4 e^{4 t}$, respectively, which is the exact solution in this example.

Remark 3. From the above two examples, it can be seen that the exact solution is obtained by using one iteration step only. 
TABLE 1: Absolute errors of $u_{n}$ at $t=0.1$ for Example 3 .

\begin{tabular}{lcccr}
\hline$x$ & $\left|u-u_{40}\right|$ & $\left|u-u_{60}\right|$ & $\left|u-u_{80}\right|$ & $\left|u-u_{100}\right|$ \\
\hline 0.0 & $1.005 E-6$ & $3.189 E-9$ & $1.011 E-11$ & $3.207 E-14$ \\
0.2 & $1.005 E-6$ & $3.189 E-9$ & $1.011 E-11$ & $3.207 E-14$ \\
0.4 & $1.005 E-6$ & $3.189 E-9$ & $1.011 E-11$ & $3.207 E-14$ \\
0.6 & $1.005 E-6$ & $3.189 E-9$ & $1.011 E-11$ & $3.207 E-14$ \\
0.8 & $1.005 E-6$ & $3.189 E-9$ & $1.011 E-11$ & $3.207 E-14$ \\
1.0 & $1.005 E-6$ & $3.189 E-9$ & $1.011 E-11$ & $3.207 E-14$ \\
\hline
\end{tabular}

TABLE 2: Absolute errors of $p_{n}$ for Example 3.

\begin{tabular}{lcccc}
\hline$t$ & $\left|p-p_{40}\right|$ & $\left|p-p_{60}\right|$ & $\left|p-p_{80}\right|$ & $2.405 E-13$ \\
\hline 0.0 & $7.542 E-6$ & $2.391 E-8$ & $7.585 E-11$ & $2.405 E-13$ \\
0.2 & $7.542 E-6$ & $2.391 E-8$ & $7.585 E-11$ & $2.405 E-13$ \\
0.4 & $7.542 E-6$ & $2.391 E-8$ & $7.585 E-11$ & $2.405 E-13$ \\
0.6 & $7.542 E-6$ & $2.391 E-8$ & $7.585 E-11$ & $2.405 E-13$ \\
0.8 & $7.542 E-6$ & $2.391 E-8$ & $7.585 E-11$ & $2.405 E-13$ \\
1.0 & $7.542 E-6$ & $2.391 E-8$ & $7.585 E-11$ & \\
\hline
\end{tabular}

Example 3. We consider the following inverse problem:

$$
\begin{gathered}
u_{t}=u_{x x}+p(t) u_{x} ; \quad 0<x<1, \quad 0<t<1, \\
u(x, 0)=x ; \quad 0 \leq x \leq 1, \\
u(0, t)=t ; \quad 0 \leq t \leq 1, \\
u(1, t)=1+t ; \quad 0 \leq t \leq 1, \\
\int_{0}^{\sqrt{t}} \sqrt{x} u(x, t) d x=\frac{2}{3} t^{7 / 4}+\frac{2}{5} t^{5 / 4}, \quad 0 \leq t \leq 1 .
\end{gathered}
$$

The true solution is $u(x, t)=x+t$ while $p(t)=1$. Let $u_{0}(x, t)=u(x, 0)=x$. According to (35), one can obtain the successive approximations $u_{n}(x, t)$ of $u(x, t)$ as follows:

$$
\begin{gathered}
u_{1}(x, t)=x+\frac{7}{4} t, \\
u_{2}(x, t)=x+\frac{7}{16} t, \\
u_{3}(x, t)=x+\frac{91}{64} t, \\
u_{4}(x, t)=x+\frac{175}{256} t, \\
\vdots
\end{gathered}
$$

And from (29), one can obtain the successive approximations of $p(t)$ as follows:

$$
\begin{aligned}
& p_{1}(t)=\frac{7}{16} \\
& p_{2}(t)=\frac{91}{64}
\end{aligned}
$$

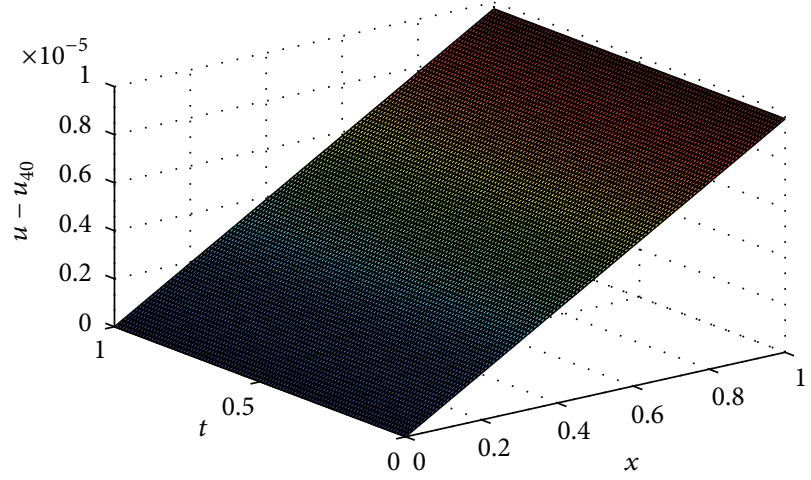

Figure 1: Graph of $\left|u(x, t)-u_{40}(x, t)\right|$ at $t=0.1$ for Example 3.

$$
\begin{gathered}
p_{3}(t)=\frac{175}{256}, \\
p_{4}(t)=\frac{1267}{1024}, \\
\vdots
\end{gathered}
$$

And the rest of the components of iteration formula (35) and (29) are obtained using the Maple Package. The obtained numerical results are summarized in Tables 1 and 2 . In addition, the graphs of the error functions $\left|u-u_{40}\right|$ and $\left|p-p_{40}\right|$ are plotted in Figures 1 and 2.

From these results, we conclude that the variational iteration method for this example gives remarkable accuracy in comparison with the exact solution. 


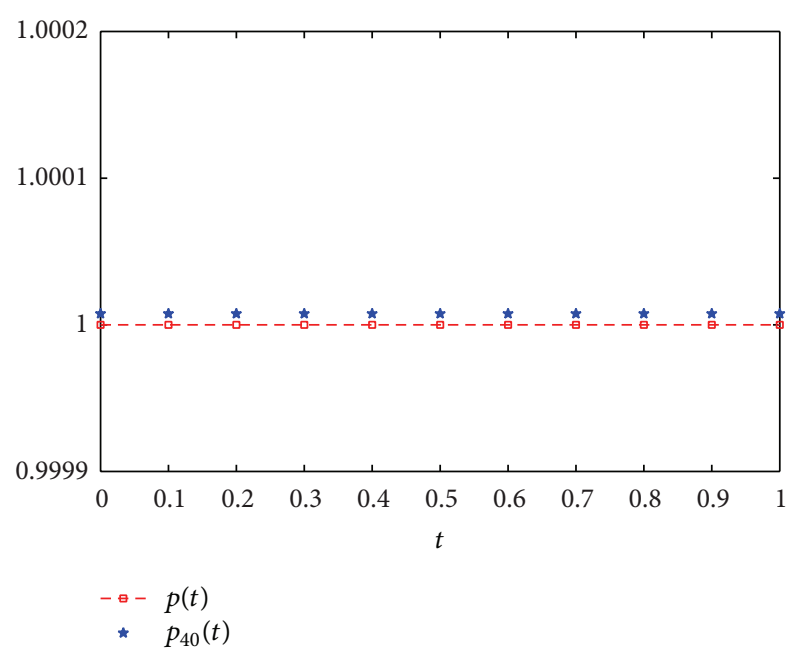

FIgURE 2: Graph of $\left|p(t)-p_{40}(t)\right|$ for Example 3.

\section{Conclusion}

In the present work, we have demonstrated the applicability of the VIM for solving a class of parabolic inverse problem in reaction-diffusion equation and introduce an estimation of the absolute error of the approximate solution for the proposed method. The method needs much less computational work compared with traditional methods and does not need discretization. The illustrative examples show the efficiency of the method. By this method, we obtain remarkable accuracy in comparison with the exact solution. Moreover, by using only one iteration step, we may get the exact solution. We expect that, for more general cases, where the inhomogeneous term, $f\left(x, y, z, t, u, u_{x}, u_{y}, u_{z}, p\right)$, has more complicated structures, the present method works well. In conclusion we mention that the VIM can be extended for similar two- and three-dimensional inverse parabolic problems subject to integral overspecification.

\section{Conflict of Interests}

The authors declare that there is no conflict of interests regarding the publication of this paper.

\section{References}

[1] M. Dehghan, "An inverse problem of finding a source parameter in a semilinear parabolic equation," Applied Mathematical Modelling, vol. 25, no. 9, pp. 743-754, 2001.

[2] J. V. Beck, B. Blackwell, and C. R. St Clair, Inverse Heat Conduction: Ill-Posed Problems, Wiley-Interscience, New York, NY, USA, 1985.

[3] W. C. Chew and Y. M. Wang, "Reconstruction of two-dimensional permittivity distribution using the distorted Born iterative method," IEEE Transactions on Medical Imaging, vol. 9, no. 2, pp. 218-225, 1990.

[4] C. Glorieux, J. Moulder, J. Basart, and J. Thoen, "Determination of electrical conductivity profiles using neural network inversion of multi-frequency eddy-current data," Journal of Physics D: Applied Physics, vol. 32, no. 5, pp. 616-622, 1999.
[5] H. W. Engl, M. Hanke, and A. Neubauer, Regularization of Inverse Problems, Kluwer Academic Publishers, Boston, Mass, USA, 1996.

[6] G. Vries, T. Hillen, M. Lewis, J. Müller, and B. Schönfisch, A Course in Mathematical Biology, SIAM, Philadelphia, Pa, USA, 2006.

[7] W. Hundsdorfer and J. Verwer, Numerical Solution of TimeDependent Advection-Diffusion-Reaction Equations, vol. 33, Springer, Berlin, Germany, 2003.

[8] J. R. Cannon and S. Perez-Esteva, "Determination of the coefficient of $\mathrm{u}_{x}$ in a linear parabolic equation," Inverse Problems, vol. 10, no. 3, pp. 521-531, 1994.

[9] J. R. Cannon and H.-M. Yin, "On a class of nonlinear parabolic equations with nonlinear trace type functionals," Inverse Problems, vol. 7, no. 1, pp. 149-161, 1991.

[10] H. Azari and F. Parzlivand, "Determination of the coefficient in the advection diffusion equation using collocation and radial basis function," International Journal of Information and Systems Sciences, vol. 1, no. 1, pp. 1-11, 2009.

[11] H. Azari, "Numerical procedures for the determination of an unknown coefficient in parabolic differential equations," Dynamics of Continuous, Discrete \& Impulsive Systems B: Applications \& Algorithms, vol. 9, no. 4, pp. 555-573, 2002.

[12] Y. P. Lin and R. J. Tait, "Finite-difference approximations for a class of nonlocal parabolic boundary value problems," Journal of Computational and Applied Mathematics, vol. 47, no. 3, pp. 335-350, 1993.

[13] J. A. MacBain, "Inversion theory for a parameterized diffusion problem," SIAM Journal on Applied Mathematics, vol. 47, no. 6, pp. 1386-1391, 1987.

[14] B. F. Jones, "Determination of a coefficient in a parabolic differential equation, I. Existence and uniqueness," Journal of Mathematics and Mechanics, vol. 11, pp. 907-918, 1962.

[15] J. Douglas and B. F. Jones, "The determination of a coefficient in a parabolic differential equation. II. Numerical approximation," Journal of Mathematics and Mechanics, vol.11, pp. 919-926, 1962.

[16] J. R. Cannon, "Determination of certain parameters in heat conduction problems," Journal of Mathematical Analysis and Applications, vol. 8, no. 2, pp. 188-201, 1964.

[17] J. Jones, "Various methods for finding unknown coefficients in parabolic differential equations," Communications on Pure and Applied Mathematics, vol. 16, pp. 33-44, 1963.

[18] J. R. Cannon and D. Zachmann, "Parameter determination in parabolic partial differential equations from overspecified boundary data," International Journal of Engineering Science, vol. 20, no. 6, pp. 779-788, 1982.

[19] J. R. Cannon and H.-M. Yin, "A class of nonlinear nonclassical parabolic equations," Journal of Differential Equations, vol. 79, no. 2, pp. 266-288, 1989.

[20] J.-H. He, "Variational iteration method for autonomous ordinary differential systems," Applied Mathematics and Computation, vol. 114, no. 2-3, pp. 115-123, 2000.

[21] E. Yusufoglu, "Variational iteration method for construction of some compact and noncompact structures of Klein-Gordon equations," International Journal of Nonlinear Sciences and Numerical Simulation, vol. 8, no. 2, pp. 153-158, 2007.

[22] J. H. He, "Approximate analytical solution for seepage flow with fractional derivatives in porous media," Computer Methods in Applied Mechanics and Engineering, vol. 167, no. 1-2, pp. 57-68, 1998. 
[23] L.-N. Zhang and J.-H. He, "Resonance in Sirospun yarn spinning using a variational iteration method," Computers \& Mathematics with Applications, vol. 54, no. 7-8, pp. 1064-1066, 2007.

[24] J.-H. He, Y.-P. Yu, J.-Y. Yu, W.-R. Li, S.-Y. Wang, and N. Pan, “A nonlinear dynamic model for two-strand yarn spinning," Textile Research Journal, vol. 75, no. 2, pp. 181-184, 2005.

[25] M. A. Abdou and A. A. Soliman, "Variational iteration method for solving Burger's and coupled Burger's equations," Journal of Computational and Applied Mathematics, vol. 181, no. 2, pp. 245251, 2005.

[26] A. A. Soliman, "A numerical simulation and explicit solutions of KDV-Burgers' and Lax's seventh-order KDV Equations," Chaos, Solitons and Fractals, vol. 29, no. 2, pp. 294-302, 2006.

[27] Z. M. Odibat and S. Momani, "Application of variational iteration method to nonlinear differential equations of fractional order," International Journal of Nonlinear Sciences and Numerical Simulation, vol. 7, no. 1, pp. 27-34, 2006.

[28] M. Inokuti, H. Sekine, and T. Mura, "General use of the Lagrange multiplier in nonlinear mathematical physics," in Variational Method in the Mechanics of Solids, S. Nemat-Nasser, Ed., pp. 159-162, Pergamon Press, Oxford, UK, 1978.

[29] J.-H. He, "Variational principles for some nonlinear partial differential equations with variable coefficients," Chaos, Solitons \& Fractals, vol. 19, no. 4, pp. 847-851, 2004.

[30] J.-H. He, "Variational iteration method-some recent results and new interpretations," Journal of Computational and Applied Mathematics, vol. 207, no. 1, pp. 3-17, 2007.

[31] A.-M. Wazwaz, "A comparison between the variational iteration method and Adomian decomposition method," Journal of Computational and Applied Mathematics, vol. 207, no. 1, pp.129136, 2007.

[32] M. Tatari and M. Dehghan, "On the convergence of He's variational iteration method," Journal of Computational and Applied Mathematics, vol. 207, no. 1, pp. 121-128, 2007. 


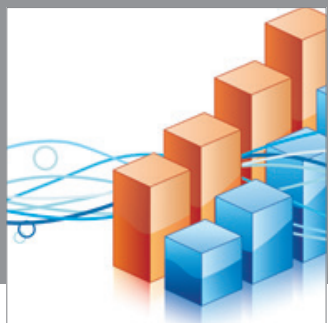

Advances in

Operations Research

mansans

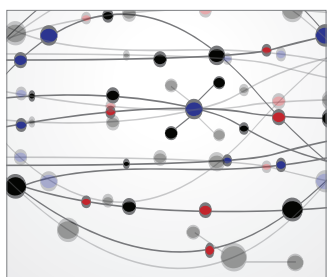

The Scientific World Journal
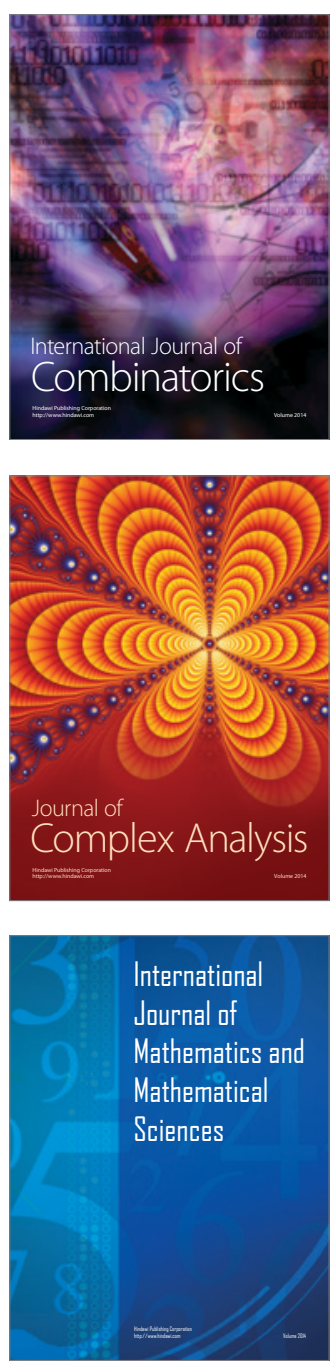
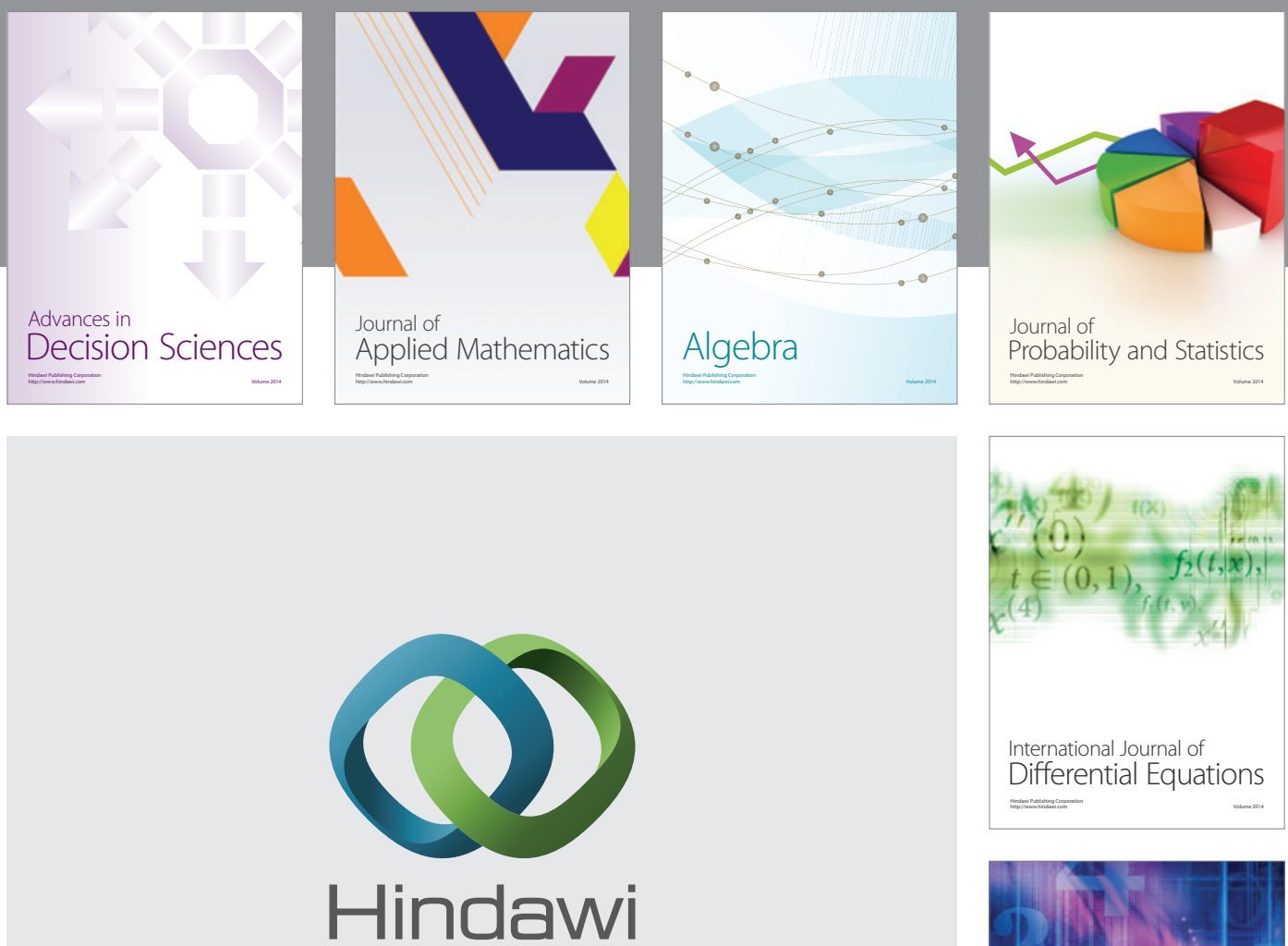

Submit your manuscripts at http://www.hindawi.com
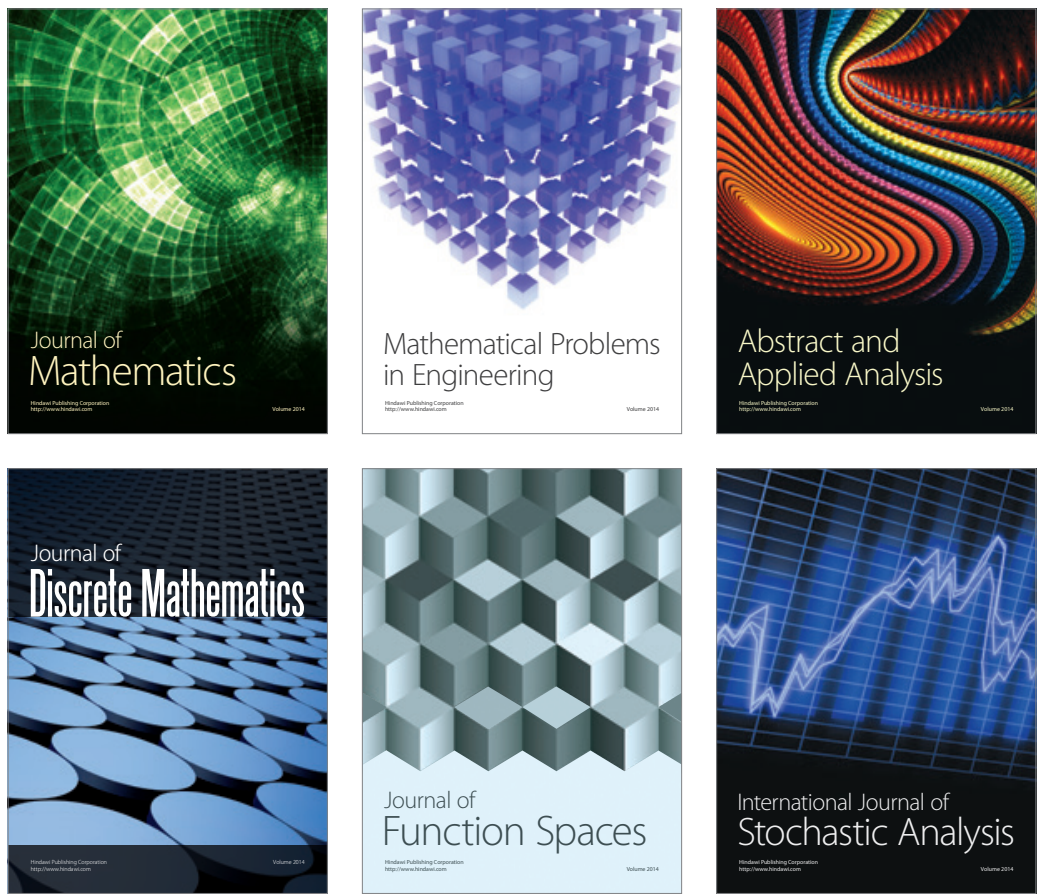

Journal of

Function Spaces

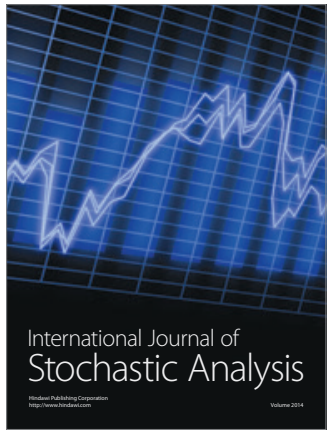

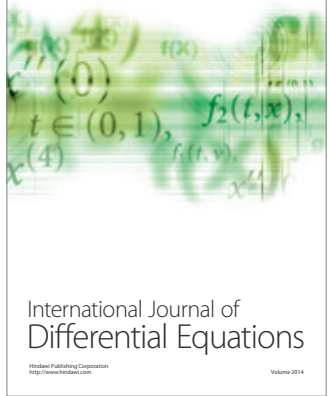
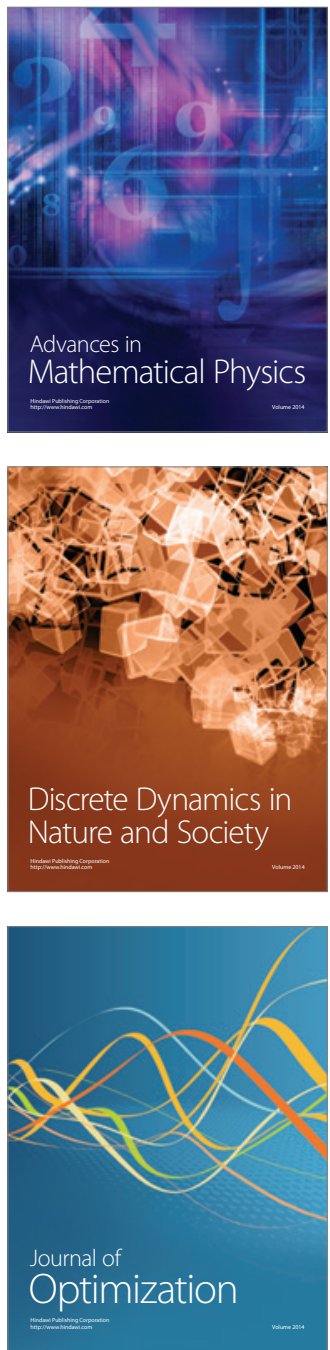